\title{
Treatment of plasmablastic lymphoma with multiple organ involvement
}

\author{
Rong Liang ${ }^{1}, \mathrm{MD}, \mathrm{PhD}$, Zhe $\underline{\text { Wang}}^{2}$, MD, PhD, Xie-qun $\underline{\text { Chen }}^{1}$, MD, PhD, Qiang-xian $\underline{B a i}^{1}$, MD, PhD
}

\begin{abstract}
We herein report the case of a 50-year-old woman who presented with persistent fever and a large mass in her right breast. Haematology, liver function, and other blood test results were abnormal. Computed tomography and positron emission tomography indicated that the lesion had spread to multiple organs. Immunohistochemical staining confirmed that the patient had plasmablastic lymphoma, an invasive and rare form of diffuse large B-cell lymphoma, and an underlying infection by the Epstein-Barr virus. After three rounds of CHOPE chemotherapy, followed by hyperCVAD and ESHAP, the patient achieved rapid and complete remission. This case is unusual in that the patient presented with a large breast mass and her recovery was extremely rapid.
\end{abstract}

Keywords: chemotherapy, CHOPE, clinicopathology, diffuse large B-cell lymphoma, plasmablastic lymphoma

\section{INTRODUCTION}

Plasmablastic lymphoma (PBL) is a highly invasive and rare form of diffuse large B-cell lymphoma (DLBCL) that most often occurs in the mouths of human immunodeficiency virus (HIV)-positive patients. ${ }^{(1,2)}$ The World Health Organization (WHO) classifies PBL into the same subtype as HIV-related non-Hodgkin lymphoma (NHL). ${ }^{(3)} \mathrm{PBL}$ is characterised by the presence of $\mathrm{B}$ lymphocytes that are terminally differentiated and have a unique immunophenotype, with expressions of VS38c, CD38, MUM1 (multiple myeloma oncogene 1) and CD138, but minimal or no expressions of CD45, CD20, CD79a and PAX5. ${ }^{(2)}$ The diagnosis and treatment are challenging and the prognosis is often poor. ${ }^{(1,2)}$ We herein report the diagnosis of a patient with $\mathrm{PBL}$, who had a large breast mass and multiple organ involvement. She was rapidly and successfully treated with chemotherapy. We also compare our case with similar reports from the literature.

\section{CASE REPORT}

In April 2012, a 50-year-old woman was admitted to our hospital due to a large mass beside the right breast and fever with no obvious cause for the last two weeks (highest temperature $>39^{\circ} \mathrm{C}$ ). Physical examination indicated a firm mass (about $10 \mathrm{~cm} \times 8 \mathrm{~cm} \times 6 \mathrm{~cm}$ ) in the upper inner quadrant of the right breast and multiple bilateral swollen lymph nodes in the neck, armpits, and groin, the largest of which was about $2 \mathrm{~cm} \times 3 \mathrm{~cm}$. The breast mass was firm and fixed with no obvious fluctuation, and the surface was red, swollen, and congested with signs of ulceration. Bone marrow puncture indicated an increased proportion of promyelocytes and myelocytes, and hyperplasia of early-stage cells in the myeloid system. Flow cytometry indicated no abnormal cell subsets, and a test for the PML/ RAR $\alpha$ fusion gene was negative. The results from chest computed tomography (CT) were suggestive of a lung infection. After admission, the patient experienced persistent fever and progressive exhaustion.

Table I shows the laboratory results. The patient's haematology and liver function tests were abnormal, and the levels of ultra-
Table I. Laboratory parameters and reference ranges.

\begin{tabular}{lcc}
\hline Parameter & Patient & Reference range \\
\hline Haematology & & \\
White blood cell count & $0.8 \times 10^{9} / \mathrm{L}$ & $3.54-9.06 \times 10^{9} / \mathrm{L}$ \\
Haemoglobin & $102 \mathrm{~g} / \mathrm{L}$ & $120-158 \mathrm{~g} / \mathrm{L}$ \\
Platelet count & $7 \times 10^{9} / \mathrm{L}$ & $165-415 \times 10^{9} / \mathrm{L}$ \\
Liver function & & \\
ALT & 26 & $5-0 \mathrm{U} / \mathrm{L}$ \\
AST & 58 & $5-40 \mathrm{U} / \mathrm{L}$ \\
Total protein & $38 \mathrm{~g} / \mathrm{L}$ & $60-80 \mathrm{~g} / \mathrm{L}$ \\
Globulin & $11.7 \mathrm{~g} / \mathrm{L}$ & $23-36 \mathrm{~g} / \mathrm{L}$ \\
Albumin & $26.3 \mathrm{~g} / \mathrm{L}$ & $35-55 \mathrm{~g} / \mathrm{L}$ \\
Alkaline phosphatase & $246 \mathrm{IU} / \mathrm{L}$ & $42-98 \mathrm{IU} / \mathrm{L}$ \\
B-microglobulin & $8.53 \mathrm{mg} / \mathrm{L}$ & $1.1-2.4 \mathrm{mg} / \mathrm{L}$ \\
Other & & \\
Ultra-sensitive CRP & $58.3 \mathrm{mg} / \mathrm{L}$ & $\mathrm{Up} \mathrm{to} 5 \mathrm{mg} / \mathrm{L}$ \\
Ferritin & $1020 \mu \mathrm{g} / \mathrm{L}$ & $12-150 \mathrm{gg} / \mathrm{L}$ \\
\hline
\end{tabular}

ALT: alanine aminotransferase; AST: aspartate transaminase; CRP: C-reactive protein

sensitive C-reactive protein and ferritin were high. The test for the Epstein-Barr virus (EBV) indicated the presence of EBV viralcapsid antigen, EBV nuclear antigen, and EBV-encoded small RNAs (EBERs). The results from the complement, rheumatoid series, and auto-antibody tests were normal, and the hepatitis B and HIV tests were negative.

Imaging by $\mathrm{CT}$ and positron emission tomography (PET) indicated a soft tissue lesion in the lower inner quadrant of the right breast, with a maximum standard uptake value (SUV) of 3.5-5.1 and a mean SUV of 2.9-3.6 (Fig. 1). Mucosal, thickened lesions of the nasopharyngeal top wall were present. Multiple lymph nodes were found around the right parotid gland and lateral to the right triceps; the nodes were found bilaterally in these regions: the neck muscle gaps; under the jaws; at the root of the neck; the supraclavicular and infraclavicular areas; the axilla; at the hila of the lungs; around the iliac artery at the level of lumbar vertebrae 5-coccyx; the iliac fossa; and the groin area. The lymph nodes were also present in the mediastinum, the right

${ }^{1}$ Department of Hematology, ${ }^{2}$ Department of Pathology, Xijing Hospital, Fourth Military Medical University, Xi'an, Shaanxi Province, China

Correspondence: Prof Rong Liang, Vice Director, Department of Hematology, Xijing Hospital, Fourth Military Medical University, No. 17, Changle West Road, Xi'an, Shaanxi Province, China, 710032. rongliang1017@gmail.com 

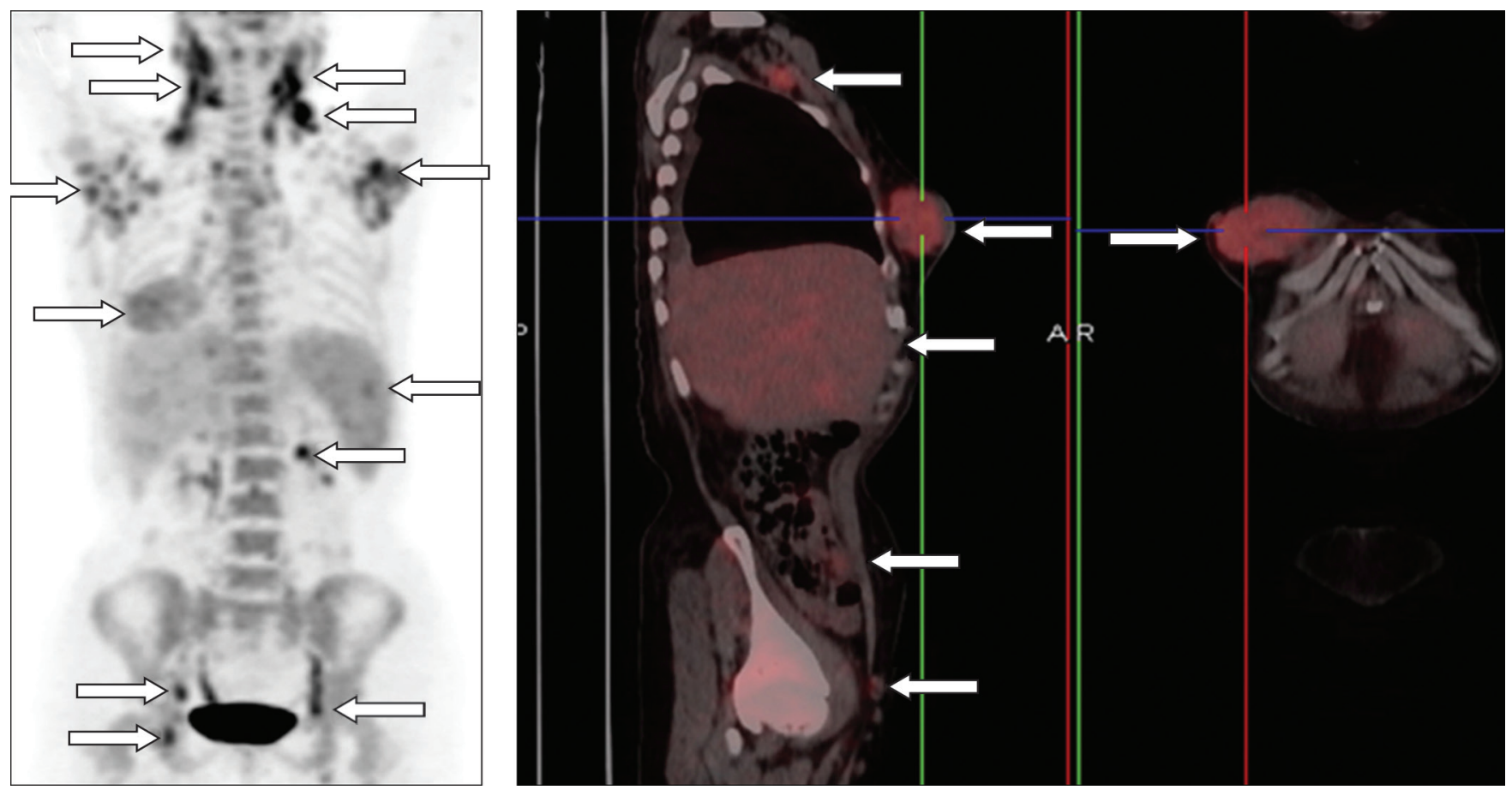

Fig. 1 CT and 18F-fluorodeoxyglucose PET images show the locations of the lesions (arrows).

third rib-sternum gap, the hilar area of the liver, the head of the pancreas-duodenum gap, and around the abdominal aorta at the level of lumbar vertebrae 1-4. PET indicated an increased glucose metabolism in all of the aforementioned locations. These results are indicative of malignant lesions, with a high probability of lymphoma.

There were several nodular and flaky isodense lesions in the liver and spleen, and nodular isodense lesions in the cortex at the lower pole of the left kidney. These lesions also had increased glucose metabolism based on PET, so we considered them as malignant with a high probability of lymphoma invasion. The spine, bilateral scapula, sternum, multiple bilateral ribs, pelvis, and bone marrow area at the proximal end of the limb bones had symmetrical and homogenously increased glucose metabolism, suggesting reactive changes.

Biopsy of the right breast tissue followed by immunostaining indicated proliferation of lymphoid tissue in the fibrous tissue and diffuse infiltration of lymphocytes and plasma cells (Fig. 2). There was no bone marrow involvement. Immunohistochemical results showed that cells were negative for CD20 and PAX5, but positive for CD79a, CD138, CD38, MUM1, Ki-67 (80\%) and EBER (Fig. 2). Additional results indicated the presence of these cells: nuclear factor kappa-light-chain-enhancer of activated B cells (NF-kB)+;

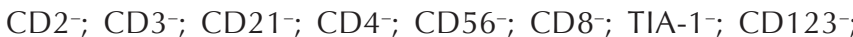
$\mathrm{ALK}^{-} ; \mathrm{CD}^{-}$; and cyclin $\mathrm{D}^{-}$(data not shown). These results are indicative of DLBCL of the PBL subtype. Immunoglobulin electrophoresis indicated overproduction of immunoglobulin G and immunoglobulin light chain lambda. Based on the presence of diffuse involvement of extralymphatic organs, we classified the patient as having stage IV type B + H (liver invasion) + D (skin and subcutaneous tissue invasion) disease, according to the Ann Arbor staging.
The patient was initially given a broad-spectrum combination of anti-infective therapy, high-dose gamma globulin, and glucocorticoid therapy. However, her body temperature, haemogram, and the size of the breast lump did not change significantly. After the diagnosis of $\mathrm{PBL}$, three courses of chemotherapy using the standard 21-day CHOPE (cyclophosphamide + doxorubicin hydrochloride [hydroxydaunorubicin], vincristine sulfate [Oncovin], prednisone, and etoposidephosphate) regimen with SULPERAZON ${ }^{\circledR} 2 \mathrm{~g}$ tid and intravenous vancomycin $0.5 \mathrm{~g}$ tid were administered. On Day 1 after initiation of chemotherapy, the patient's body temperature returned to normal and the breast mass and superficial lymph nodes were significantly smaller. The haemogram returned to normal a few days later. The patient was given two further cycles consisting of hyperCVAD (cyclophosphamide, mesna, vincristine, adriamycin) and then ESHAP (etoposide, cisplatin, methyprednisolone, cytarabine). After 28 days, another round of CHOPE, hyperCVAD, and ESHAP were given. The rationale for switching regimens was to prevent development of drug resistance, because the patient was at high risk and her NHL subtype was indicative of poor prognosis.

Chest CT performed in February 2013 indicated a $1 \mathrm{~mm} \times$ $1 \mathrm{~mm}$ lesion, with mild bilateral pleural effusion but no enlarged hilar or mediastinal lymph nodes. As of October 2013, the patient still had lesions, but there was no disease progression and the patient was otherwise healthy with no signs of recurrence. Abdominal ultrasonography indicated reduced density of nodular, flaky lesions in the liver and spleen with no enlarged lymph nodes. The patient could not afford a follow-up PET or CT examination. Although the use of $\mathrm{CHOPE}$ regimen for treatment of $\mathrm{PBL}$ is unremarkable in itself, this case is unusual because the patient presented with a large breast mass and her recovery was extremely rapid. 

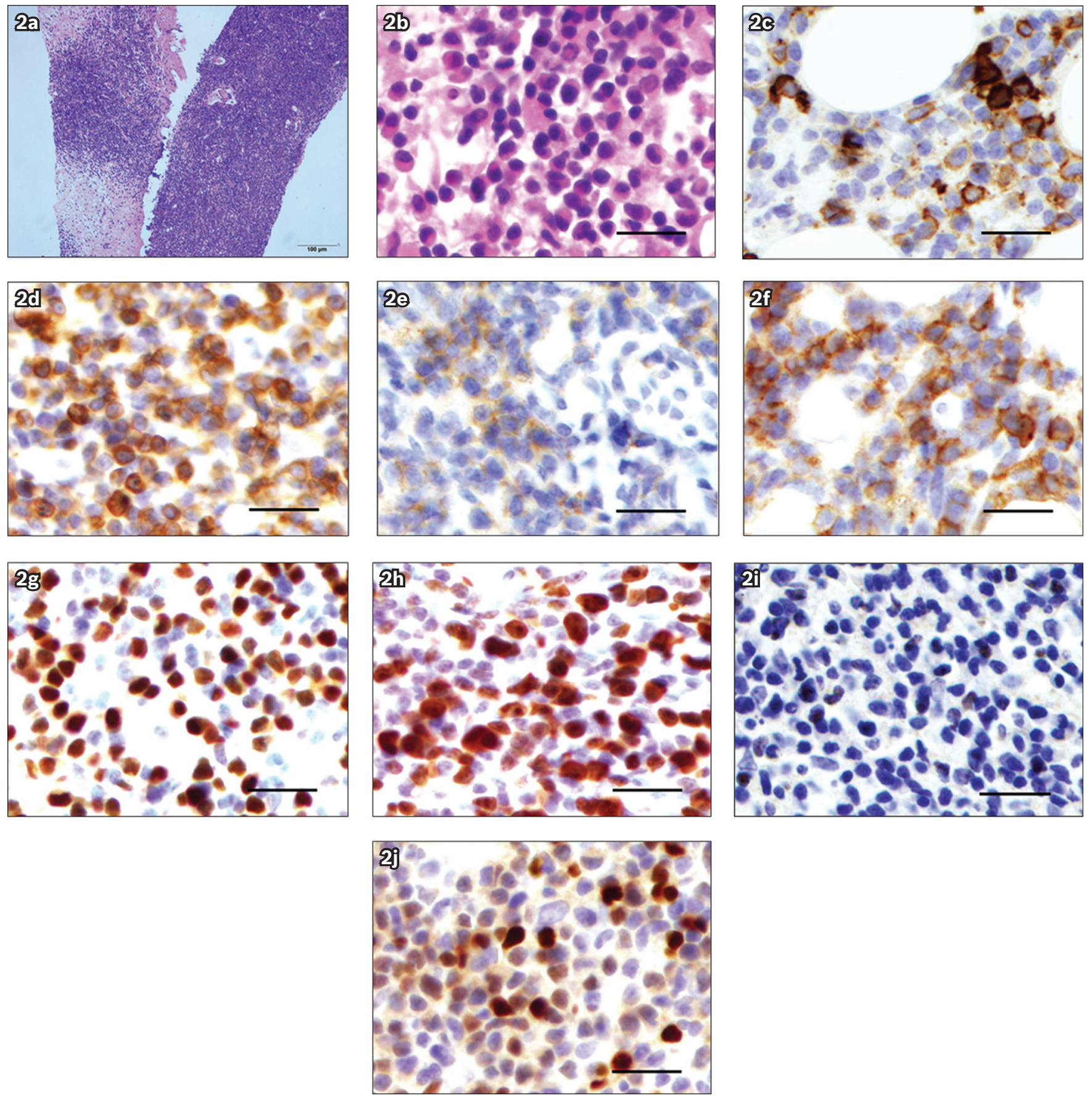

Fig. 2 Photomicrographs show the tumour under (a) low magnification and (b) high magnification on haematoxylin \& eosin staining; and immunohistochemical staining for (c) CD20; (d) CD79a; (e) CD138; (f) CD38; (g) MUM1; (h) Ki-67; (i) EBER; and (j) PAX5. The scale bar is $50 \mu \mathrm{m}$, and the antibody source and catalogue numbers are as follows: anti-CD20, DAKO IR604; anti-CD79, DAKO IR621; anti-CD138, DAKO IR642; anti-CD38, SANTA CRUZ sc7325; anti-MUM1, DAKO IR644; anti-KI67, DAKO IR626; and anti-PAX-5, DAKO IR650.

\section{DISCUSSION}

PBL is a rare lymphoma that was first described by Delecluse et al in 1997.(1) The 2008 WHO classification considers PBL as an independent disease that is a subtype of DLBCL. ${ }^{(3)} \mathrm{PBL}$ has a high incidence in HIV-positive patients and is more common in men. ${ }^{(2)} \mathrm{PBL}$ also seems to be more common in patients who have weakened immune systems due to infection by EBV or human herpes virus 8 . Castillo et al, ${ }^{(4)}$ who described the greatest number of PBL cases $(n=228)$, reported that $69 \%$ of patients (mean age $=$ 39 years) were HIV-positive, and that most HIV-negative patients (mean age $=58$ years) were EBV-positive. EBV infection can be confirmed by the measurement of EBER. Our female patient was HIV-negative but EBER-positive, supporting the hypothesis that viral infection has a role in the pathogenesis of PBL.

PBL lesions are most common in the mouths and gastrointestinal tracts of HIV-positive patients. Extranodal lymphoid organs, the central nervous system, peripheral lymph nodes, paranasal sinuses, mediastinum, lung, liver, and bone marrow may also be involved. ${ }^{(2)}$ There have been recent reports of PBL in HIVnegative patients in whom the lesions mostly occurred in the extranodal tissues, including the skin, soft tissue, maxillary sinus, and gastrointestinal tract. ${ }^{(5,6)}$ 
There has been only one previous report of a simple breast PBL lesion. ${ }^{(7)}$ The initial symptoms in our patient were a large breast lump and fever, and these were followed by clinical manifestations of multiple organ involvement including the nasopharynx, systemic multiple lymph nodes, liver, and spleen. Our patient was HIV-negative and classified as stage IV type $\mathrm{B}+\mathrm{H}+\mathrm{D}$ PBL. ${ }^{(8)}$ This is the first reported case of PBL in which the initial symptom was a large breast lump and the patient had multiple organ involvement.

The PBL cells in our patient were atypical medium and large lymphocytes that had round, oval, or irregular shaped nuclei with one or more clear nucleoli. The chromatin appeared to be normal, and the cells had a moderate or abundant amount of cytoplasm, with no evidence of a 'starry' appearance. Immunophenotyping is critical for the diagnosis of PBL. (2) Tumour cells from our patient had the morphology of plasma cells and immunochemical properties consistent with PBL (Fig. 2).

$\mathrm{PBL}$ is highly malignant and invasive, associated with poor prognosis, and has low sensitivity to chemotherapy. There is currently no standard chemotherapy protocol, and most regimens are based on the $\mathrm{CHOP}$ regimen. A recent international multicentre analysis of HIV-positive cases with PBL showed that intense chemotherapy did not extend survival time; the rate of complete remission (CR) was $66 \%$ and the median overall survival was 11 months. ${ }^{(9)}$ A recent single-centre study of nine HIV-negative PBL patients who received chemotherapy (CHOP or hyperCVAD) reported that seven patients achieved CR and one patient achieved partial remission. ${ }^{(10)}$ In addition, four of these patients underwent autologous peripheral blood haematopoietic stem cell transplantation during the first CR. Seven patients were alive at the follow-up (median $=23.9$ months). ${ }^{(10)}$ Although only a small number of PBL cases have been examined, PBL seems to have a high degree of malignancy and associated with a poor prognosis relative to NHLs.

Our patient underwent four courses of chemotherapy using the $\mathrm{CHOPE}$ regimen, and at 18 months after presentation, she had fully recovered from $\mathrm{PBL}$, although recurrence is still possible. The patient's tumour cells tested positive for nuclear expression of NF- $\kappa B$, suggesting that a protocol using bortezomib, a novel proteasome inhibitor, may be effective. A recent report indicated that bortezomib alone was successful in the treatment of an HIV-positive patient with PBL. ${ }^{(11)}$ In view of our past experience treating refractory mantle cell lymphoma with PAD (bortezomib + doxorubicin + dexamethasone), ${ }^{(12)}$ we plan to consider the use of $\mathrm{PAD}$ regimen for future treatment of $\mathrm{PBL}$. There are currently no standard prognostic indicators of PBL. However, a recent report suggested that quantitation of the EBV DNA may be useful for assessment of the response to treatment. ${ }^{(13)}$ Accordingly, we suggest quantitative detection of EBV to be performed in clinical practice and that new drug regimens, such as PAD, should be actively tested in order to improve the prognosis of patients diagnosed with PBL.

\section{REFERENCES}

1. Delecluse HJ, Anagnostopoulos I, Dallenbach F, et al. Plasmablastic lymphomas in the oral cavity: a new entity associated with the human immunodeficiency virus infection. Blood 1997; 89:1413-20.

2. Castillo JJ, Reagan JL. Plasmablastic lymphoma: a systematic review. ScientificWorldjournal 2011; 11:687-96.

3. Stein H, Harris N, Campo E. Plasmablastic lymphoma. In: Swerdlow S, Campo E, Harris N, Jaffe E, Pileri S, Stein H, Thile J, Vardiman J, eds. WHO classification of tumours of the haematopoietic and lymphoid tissues. Lyon France: IARC, 2008:256-57.

4. Castillo JJ, Winer ES, Stachurski D, et al. Clinical and pathological differences between human immunodeficiency virus-positive and human immunodeficiency virus-negative patients with plasmablastic lymphoma. Leuk Lymphoma 2010; 51:2047-53

5. Tavora F, Gonzalez-Cuyar LF, Sun CC, Burke A, Zhao XF. Extra-oral plamablastic lymphoma: report of a case and review of literature. Hum Pathol 2006; 37:1233-6.

6. Mansoor M, Alani FS, Aslam MB, et al. A case report of cecal plasmablastic lymphoma in a HIV-negative patient. Eur J Gastroenterol Hepatol 2012; 24:332-5.

7. Wang J, Hermandez OJ, Sen F. Plasmablastic lymphoma involving breast: a case diagnosed by fine-needle aspiration and core needle biopsy. Diagn Cytopathol 2008; 36:257-61.

8. Carbone PP, Kaplan HS, Musshoff K, Smithers DW, Tubiana M. Report of the Committee on Hodgkin's Disease Staging Classification. Cancer Res $1971 ; 31: 1860-1$.

9. Castillo JJ, Furman M, Beltrán BE, et al. Human immunodeficiency virusassociated plasmablastic lymphoma: poor prognosis in the era of highly active antiretroviral therapy. Cancer 2012; 118:5270-7.

10. Liu JJ, Zhang L, Ayala E, et al. Human immunodeficiency virus (HIV)negative plasmablstic lymphoma: a single institutional experience and literature review. Leuk Res 2011; 35:1571-7.

11. Bibas M, Grisetti S, Alba L, et al. Patient with HIV-associated plasmablastic lymphoma responding to bortezomib alone and in combination with dexamethasone, gemcitabine, oxaliplatin, cytarabine, and pegfilgrastim chemotherapy and lenalidomide alone. J Clin Oncol 2010; 28:e704-8.

12. Liang R, Chen XQ, Bai QX, et al. Successful treatment of refractory mantle cell lymphoma with PAD regimen: a case report. Chin J Cancer Prev Treat $2011 ; 18: 968-9$.

13. Law MF, Poon WL, Ng KS, et al. Quantification of plasma Epstein-Barr virus DNA for assessing treatment response in a patient with plasmablastic lymphoma. Ann Hematol 2012; 91:789-91. 